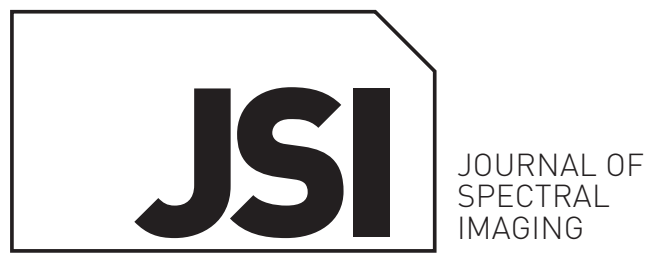

Paper Presented at HSI 2014, Hyperspectral Imaging and Applications, Coventry, UK

\title{
openaccess
}

\section{Hyperspectral to multispectral imaging for detection of tree nuts and peanut traces in wheat flour}

\author{
Puneet Mishra, ${ }^{\mathrm{a},{ }^{*}}$ Ana Herrero-Langreo, ${ }^{\mathrm{b}}$ Pilar Barreiro, ${ }^{\mathrm{a}}$ Jean Michel Roger, ${ }^{\mathrm{b}}$ Belén Diezma, ${ }^{\mathrm{a}}$ Nathalie Gorretta ${ }^{\mathrm{b}}$ and \\ Lourdes Lleóa \\ ${ }^{\circledR}$ Department of Ingeniería Rural, ETSI Agrónomos, Avda. Complutense s/n Universidad Politécnica de Madrid (UPM), CEI Moncloa, 28040 \\ Madrid, Spain. E-mail: puneet.mishra@alumnos.upm.es \\ bIrstea, UMR ITAP, 361 Rue J.F. Breton, 34196 Montpellier Cedex 5, France
}

\begin{abstract}
In current industrial environments there is an increasing need for practical and inexpensive quality control systems to detect the foreign food materials in powder food processing lines. This demand is especially important for the detection of product adulteration with traces of highly allergenic products, such as peanuts and tree nuts. Manufacturing industries dealing with the processing of multiple powder food products present a substantial risk for the contamination of powder foods with traces of tree nuts and other adulterants, which might result in unintentional ingestion of nuts by the sensitised population. Hence, the need for an in-line system to detect nut traces at the early stages of food manufacturing is of crucial importance. In this present work, a feasibility study of a spectral index for revealing adulteration of tree nut and peanut traces in wheat flour samples with hyperspectral images is reported. The main nuts responsible for allergenic reactions considered in this work were peanut, hazelnut and walnut. Enhanced contrast between nuts and wheat flour was obtained after the application of the index. Furthermore, the segmentation of these images by selecting different thresholds for different nut and flour mixtures allowed the identification of nut traces in the samples. Pixels identified as nuts were counted and compared with the actual percentage of peanut adulteration. As a result, the multispectral system was able to detect and provide good visualisation of tree nut and peanut trace levels down to $0.01 \%$ by weight. In this context, multispectral imaging could operate in conjuction with chemical procedures, such as Real Time Polymerase Chain Reaction and Enzyme-Linked Immunosorbent Assay to save time, money and skilled labour on product quality control. This approach could enable not only a few selected samples to be assessed but also to extensively incorporate quality control surveyance on product processing lines.
\end{abstract}

Keywords: hyperspectral, multispectral, near infrared (NIR), index, chemometrics, nuts, allergy, adulteration

\section{Introduction}

A nut is a fruit composed of a hard shell and an edible seed. Tree nuts (TNs) are usually used to describe a wide range of seeds except peanuts, mostly from trees, with a tough seed coat or shell. With high-energy content and positive global health impact, TNs are nutrients that have been present for centuries in the human diet. Nuts in general are concentrated sources of dietary energy, as well as sources of unsaturated fatty acids, various micronutrients and fibre. ${ }^{1}$ However, over recent decades they have increasingly been found to induce adverse health effects with allergenic reactions. The need for recognition of allergies has been rising in the industrialised world since the middle of the last century. In addition, the prevalence of food allergies has grown rapidly over the past 10 years. ${ }^{2}$ Peanuts and TNs are common food allergens and are 
the leading cause of fatalities from food-induced allergenic reactions. ${ }^{2}$ Although peanut is the most common cause of nut allergy, peanut-allergic patients are frequently also sensitive to TNs. ${ }^{3}$ Hence, the avoidance or a restricted consumption of other TNs should be recommended to peanut-sensitised individuals. $^{4}$

With an increasing number of affected individuals, allergies to nuts are a growing global concern. Allergenic conditions arising from the consumption of nuts are generally thought to be life-long and typically severe. ${ }^{5}$ No definite treatment is yet available for nut and seed allergies. ${ }^{6}$ The major allergenic compounds in TNs, like peanut and other seeds, have been identified as the seed storage proteins. ${ }^{7}$ Accordingly, analytical protein detection methods are commonly used by the food industry and official food control agencies for the detection of allergens. The two commonly used methods are Enzyme-Linked Immunosorbent Assay (ELISA) ${ }^{8}$ and Real Time Polymerase Chain Reaction (RT PCR). ${ }^{9}$ However, these methods are expensive, time-consuming and require chemical expertise, which makes their implementation difficult in food manufacturing processes.

Dietary avoidance is the primary way to manage these allergies and requires the ability to identify peanuts or tree nuts. ${ }^{10}$ However, manufacturing industries dealing with the processing of multiple powder food products present a substantial risk for the contamination of powder foods with TN traces and other adulterants. Such cross contamination might result in unintentional ingestion of nuts by the sensitised population. The need for an in-line system to detect nut traces at the early stages of food manufacturing is thus crucial to both the nutallergic population and to food manufacturers.

Various applications of hyperspectral imaging (HSI), such as detection of infestation in soybean ${ }^{11}$ and melamine in milk powders, ${ }^{12}$ have illustrated the suitability of this technology for the detection of adulterants in powder foods. Nevertheless, the high cost and complexity of HSI, together with its large data-storage requirements, represents an important limitation to its industrial application. ${ }^{13}$ In this context, feasibility studies carried out using complete spectral information are commonly a first step for further simplification into multispectral technology. HSI can be used to determine the optimal wavebands for a particular application. This approach allows simplification of image acquisition and processing. By avoiding a long sequence of scans in the spectral domain, multispectral systems provide faster image acquisition and simpler algorithms for image processing and decision making. ${ }^{14}$ Some recent work demonstrating the feasibility of multispectral systems are the supervision of quality deterioration in ready-to-use leafy spinach during storage, ${ }^{15}$ the comparison of multispectral indexes related to maturity in peaches, ${ }^{16}$ non-destructive detection of transgenic rice seeds, ${ }^{17}$ non-destructive determination of aerobic plate count (APC) in cooked pork sausages, ${ }^{18}$ mitosis detection in cancer cells with multispectral imaging ${ }^{19}$ and detection of frass contamination on mature Campari tomatoes. ${ }^{20}$ In these studies, multispectral imaging systems proved to be an effective alternative to the traditional analytical procedures, offering cost savings and being easier to apply in an industrial context.

Regarding the detection of nut contamination, in our previous work ${ }^{21,22}$ we proposed and compared several spectral indices to discriminate peanut spectra from other powder food signatures (wheat flour, skimmed milk powder and cocoa powder). All indices were based on a spectral band at $1200 \mathrm{~nm}$, associated with the presence of lipids in peanuts. Other surrounding wavebands were integrated in the indices to improve their robustness towards unrelated variations of the spectra and product modalities. These indices were tested on pure spectra using a point contact spectrophotometer; the spectral index (SI) obtained good results with clear discrimination of the pure products. The SI was effective in correctly identifying peanut spectra regardless of their origin and roasting treatments.

In a practical environment, spectral imaging is better adapted to industrial applications than plain spectroscopy. It can be implemented to assess moving processing lines, it allows complete sampling of the product going through the industrial process and it is able to observe the spatial distribution of the adulterant particles on the line. In this context, the present work aims to be a step forward from point contact spectroscopy towards multispectral imaging. The objective of this research is to study the feasibility of a previously developed ${ }^{21,22}$ spectral index for revealing adulteration of TNs and peanuts traces in wheat flour samples on hyperspectral images. Several nuts: peanut, walnut and hazelnut, with similar lipid profiles and potential hazardous properties for sensitised individuals are included in this study. The SI developed through point contact spectroscopy is applied to hyperspectral images obtained for different percentages of adulterated flour samples with different nut traces. Also, a mathematical interpretation of this index relating it to the second derivative is developed and provided for better understanding.

\section{Materials and methods Tree nuts and peanuts adulterated sample preparation}

Wheat flour (Triticum aestivum), "Coeur de Blé" from the producer MasterChef, ground walnut (Juglans regia) and hazel nut (Corylus avellana) from "maître prunille" were purchased from a local supermarket in Montpellier, France. Ground peanuts (Arachis hypogaea) (IRMM-481 kit) were obtained from the European Commission Joint Research Centre, Institute for Reference Materials and Measurements. The kit (IRMM-481) with six different vials contains non-salted peanut powder with a normal particle size from $500 \mu \mathrm{m}$ to $1000 \mu \mathrm{m}$. Four of the six vials-i.e. IRMM-481a, originating from Runners, Argentina, corresponded to blanched peanuts air-roasted at $140^{\circ} \mathrm{C}$ for $20 \mathrm{~min}$; IRMM-481b originating from Common Natal, South Africa refers to raw peanuts, air-roasted at $160^{\circ} \mathrm{C}$ for $13 \mathrm{~min}$; IRMM-481c originating from Virginia, USA were 
blanched peanuts, oil roasted at $145^{\circ} \mathrm{C}$ for $25 \mathrm{~min}$; and IRMM481 e originating from Jumbo Runners, USA, were blanched peanuts without roasting-were mixed to make trace samples with wheat flour.

Three sets of nine different samples were prepared: eight samples of each set corresponded to homogeneous mixes of flour with various percentages of peanut, walnut and hazelnut adulteration separately, ranging from $10 \%$ to $0.01 \%$ by weight, making 24 samples. The remaining sample of each set corresponded to a $50: 50 \%$ mixture of pure peanut, pure walnut or pure hazelnut together with pure wheat flour, making three extra samples. 27 samples were measured in total. A KERN 770 analytical balance with a least count of $0.00001 \mathrm{~g}$ was used to weigh the adulterants. Peanut, hazelnut and walnut traces $(500-1000 \mu \mathrm{m})$ were mixed manually with the help of wooden spatulas to form a homogeneous mixture with wheat flour. New wooden spatulas were used for every sample. Two adapted aluminium platforms with an exposed sample surface area of $36 \mathrm{~cm}^{2}$ (for samples down to $0.1 \%$ adulteration) and $95 \mathrm{~cm}^{2}$ (for samples with $0.05-0.01 \%$ adulteration) were designed to present the sample for image acquisition. The depth of the surface used for sample presentation was $3 \mathrm{~mm}$. The suitability of this depth was decided by performing trials with different depths, such as $1 \mathrm{~mm}$ and $5 \mathrm{~mm}$. Depths lower than $3 \mathrm{~mm}$ were insufficient to spread and smooth the sample homogeneously because of the cohesion force between the particles. Whereas, depths of more than $3 \mathrm{~mm}$ needed more sample to enable detection of the desired concentration. Aluminium was chosen as a material because it is optically neutral, i.e., it presents constant absorption throughout the used spectral range. This property allows the spectra of the samples to be measured without interference from the platform. Samples were spread and smoothed with the help of disposable wooden spatulas for even distribution.

\section{Camera setup for hyperspectral imaging}

The images were acquired with a line-scan push broom hyperspectral imaging system. The system consists of a HySpex SWIR-320m-e hyperspectral push broom camera (Norsk Elektro Optikk, Lørenskog, Norwayl, translation stage operated by a stepper motor and a computer supported by Norsk ground software (Norsk Elektro Optikk, Lørenskog, Norway). An illumination unit including one line $150 \mathrm{~W}$ tungsten halogen source mounted at a $45^{\circ}$ angle on the side of the camera in respect to the vertical plane was used for illuminating the sample.

The translation stage moves the object along the track, while the push broom imaging system records image lines across the track. The hyperspectral camera covers the spectral range from $1000 \mathrm{~nm}$ to $2500 \mathrm{~nm}$ in 250 spectral bands (spectral resolution of $6 \mathrm{~nm}$ ) with 320 pixels over the cross-track field of view (FOV). The camera was equipped with a $30-\mathrm{cm}$ lens that produced a nominal pixel size of approximately $0.287 \mathrm{~mm}$ across and $0.427 \mathrm{~mm}$ along the FOV. Setting of acquisition parameters (integration time and dark current subtraction), data acquisition and recording were performed by means of the software provided by NEO. Prior to acquisition of images the halogen bulb was switched on for half an hour in order to stabilise the light source temperature drift and improve the spatial lightening uniformity of the sample placed under the $\mathrm{FOV}^{23}$

Samples were successively placed on the translation stage to be scanned line by line by the imaging sensors. Raw images were first corrected in radiance using sensor characteristics (e.g. spectral sensitivity) provided by the manufacturer. To obtain reflectance images (images independent of illumination conditions), a two-point reflectance calibration was performed using a white diffuse reflectance standard (Spectralon ${ }^{\circledR}$, Labsphere, France).

\section{Multispectral index as an estimate of second derivative}

The multispectral index was designed as a second order difference operator to provide an estimate for second derivative. The mathematical explanation follows:

Suppose that a function $f(x)$ is given at equally spaced discrete points, say $x_{0}, x_{1}, \ldots x_{n}$ as $f_{0}, f_{1}, \ldots f_{n}$ respectively. Also, let the constant difference between two consecutive points of $x$ be called the interval of differencing or the step length denoted by $h$. Then the difference operator is given by:

$$
\nabla_{h}[f](x)=f(x)-f(x-h)
$$

The derivative of a function given by two different consecutive point is given by:

$$
f^{\prime}(x)=\lim _{h \text { tends to } 0} \frac{f(x)-f(x-h)}{h}
$$

Now, if $h$ has a (non-zero) value instead of approaching zero, then the right-hand side of the above equation would be written as:

$$
\frac{f(x)-f(x-h)}{h}=\frac{\nabla_{h}[f](x)}{h}
$$

So, for small value of $h$

$$
f(x)-f(x-h)=\nabla_{h}[f](x)
$$

Similarly for second derivative estimate, by using the above difference formula for $f^{\prime}(x)$ and $f^{\prime}(x-h)$, i.e. Equation (1) and applying a difference formula for the derivative of $f^{\prime}$ at $x$, we obtain the difference approximation of the second derivative of $f$ :

$$
f^{\prime \prime}(x) \approx \frac{\nabla_{h}^{2}[f](x)}{h}=\frac{f(x+h)-2 f(x)+f(x-h)}{h}
$$

So again, for small value of $h$

$$
f(x+h)-2 f(x)+f(x-h)=\nabla_{h}^{2}[f](x)
$$

Hence, similarly, for the $n^{\text {th }}$ derivative estimation:

$$
\nabla_{h}^{n}[f](x)=\sum_{i=0}^{n}(-1)^{i}\left(\begin{array}{l}
n \\
i
\end{array}\right) f(x-i h)
$$


As the peanut and TNs are rich in long chain fatty acids, the absorbance spectra provided a distinct peak at $1200 \mathrm{~nm}$ due to a $\mathrm{CH}_{2}$ second overtone. ${ }^{24}$ Hence, the second derivative around this peak can help in highlighting the peanut and other TNs over the wheat flour. Two similar wavelengths at the end of the peak were taken, i.e. $1140 \mathrm{~nm}$ and $1254 \mathrm{~nm}$, as obtained by Cuadrado et al., ${ }^{21,22}$ i.e. $1141 \mathrm{~nm}$ and $1250 \mathrm{~nm}$ in their index.

Using Equation (2) for the selected wavelengths over the curve, we get

$$
f^{\prime \prime}(x) \approx \frac{\nabla_{h}^{2}[f](x)}{h}=\frac{P_{1254}-2 P_{1206}+P_{1140}}{h}
$$

when $h$ is small, Equation (5) will reduce to:

$$
\nabla_{h}^{2}[f](x)=P_{1254}-2 P_{1206}+P_{1140}
$$

Equation (6) is second order difference operator providing an estimate for second derivative over the selected wavelength region.

\section{Data analysis}

All computations and data analysis were performed with the software Matlab R2012b (The Mathworks Inc., Natick, MA, USA). As a first step, the raw hyperspectral images were converted to absorbance $\left(-\log _{10}\right)$ and reduced to multispectral planes by selecting the spectral plane corresponding to the wavelength values used in the SI (Equation 6).

$$
\text { Spectral Index }(S I)=S_{1140}+S_{1254}-2 S_{1206}
$$

Using these planes, an indexed image was obtained by computing the SI for each pixel of the image. Then, index images resulting from the images of 50:50\% pure flour with peanut, flour with walnut and flour with hazelnut traces separately were used to set up the index threshold between flour and peanut, walnut and hazelnut pixels. As the existence of only two classes require a single threshold value, therefore, this threshold was applied for segmentation on the score images of the adulterated samples. From the segmented images the percentage of pixels identified as peanut, walnut and hazelnut were counted and compared with the actual percentage of adulteration.

In order to validate the ability of the proposed index to enhance the contrast between nuts and wheat flour, the index was computed on a simulated hyperspectral image

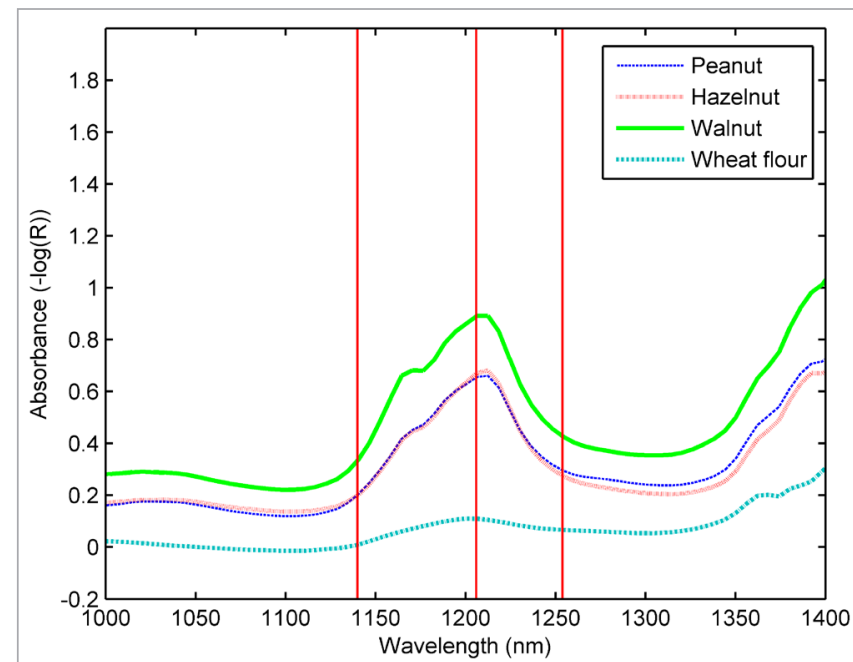

Figure 2. Mean absorbance spectra of wheat flour (blue), peanut (red), hazelnut (green) and walnut (sky blue). Vertical lines respresent the wavelengths used in the SI.

$(100 \times 300 \times 256)$, where peanut, walnut and hazelnut spectra were placed in three known positions inside the image, on a background of flour spectra. Figure 1 illustrates the main steps of processing performed for the final detection of nut traces.

\section{Results and discussion Spectral profiles}

Figure 2 plots the average absorbance spectra obtained from HSI of pure peanut, walnut, hazelnut and wheat flour. All nuts present higher absorbance than wheat flour. Within the nuts, the highest absorbance levels are observed with walnut and the lowest with hazelnut and peanut spectra. A distinct absorbance peak is found at $1200 \mathrm{~nm}$ in the spectra of peanut, walnut and hazelnut. This peak (1200 nm) can be explained by the presence of long chain fatty acids in all three nuts, associated to a $\mathrm{CH}_{2}$ second overtone absorbance peak near $1200 \mathrm{~nm} .{ }^{24}$ These spectral profiles are similar to those observed between plain spectra of peanut and wheat flour by Cuadrado et al. ${ }^{21,22}$ Consequently, the SI proposed on the latter work is suitable to discriminate all three types of nuts from wheat flour samples. Vertical lines plotted in Figure 2 indicate

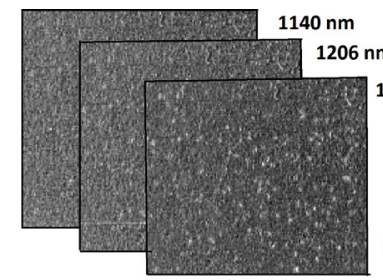

a. Planes corresponding to Index

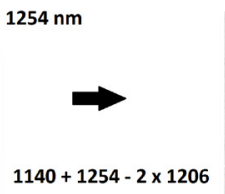

b.

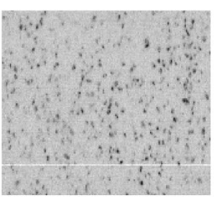

c. Score Image

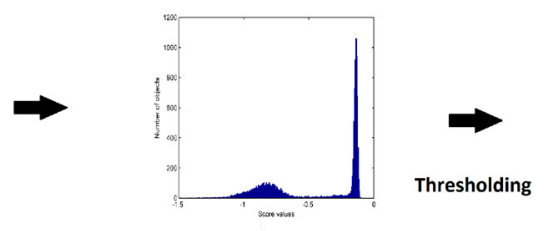

d. Histogram for selecting threshold

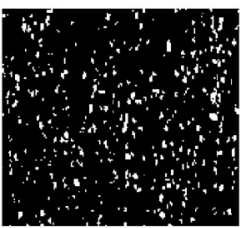

e. Classification image

Figure 1. Main steps involved in the detection of peanut and TN traces in wheat flour. (a) Selection of planes, (b) index computation, (c) index image, (d) threshold setting according to the histogram and (e) classification image used for calculating the adulteration percentage. 


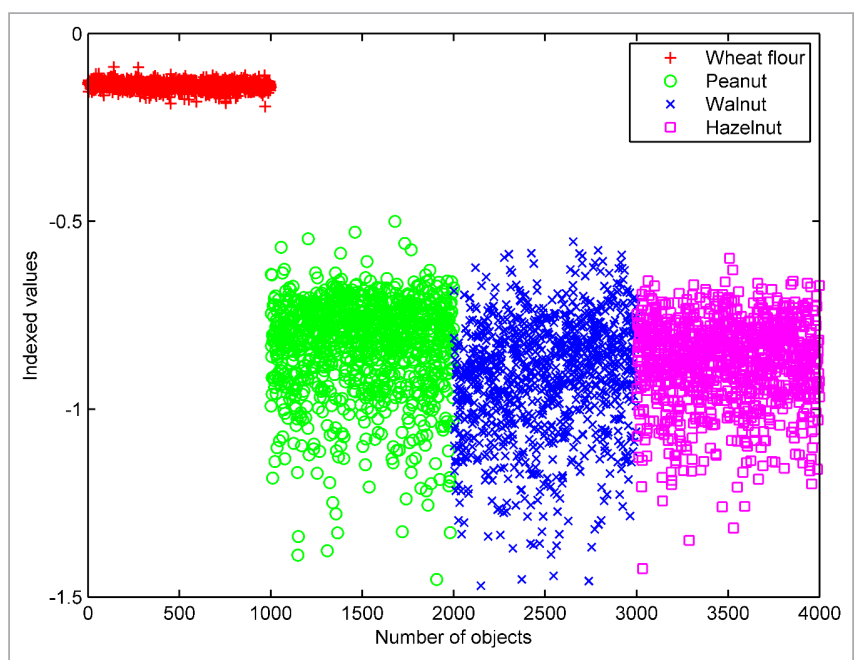

Figure 3. SI values for pure wheat flour, pure peanut, pure walnut and pure hazelnut, ( $N=1000$ pixels per product $)$.

the selected wavelengths used for the index computation. The fatty acid absorbance peak at $1200 \mathrm{~nm}$ is used in combination with two additional wavelengths, at the beginning and end of the peak, which also allowed additive effects on the spectra to be corrected by performing an estimation of second order derivative as explained above.

\section{Index discrimination ability per product and index validation}

The SI was tested with images of different pure nuts and pure wheat flour samples and corresponding index values were obtained. Index values of 1000 randomly selected pixels of pure wheat flour, peanut, walnut and hazelnut are presented in Figure 3. The representation was carried out in order to observe the discrimination power of the index. It can be seen in Figure 3 that SI values obtained for the wheat flour, ranging approximately from 0.5 to 0.6 , were clearly separated from all types of TNs and peanut. SI values for TNs and peanut lie in a common range between -0.6 and 0 . In Figure 3 , it can be seen that wheat flour possesses low variability as compared to nut score values. This could be due to the smaller particle size of wheat flour (125-100 $\mu \mathrm{m}$ and 212-160 $\mu \mathrm{m})$ as compared to that of the nuts $(500-1000 \mu \mathrm{m})$. Also, the smaller particle size of the wheat flour produces very high scattering of the light resulting in lower absorbance values, which is a reason for wheat flour being white as compared to the other products. The representation shows the ability of the index to discriminate all three kinds of nuts from wheat flour. This approach allows further implementation in a multispectral imaging system. However, the index was not designed to identify the kind of nuts. In this regard, even though the SI is not product specific, it would allow detection of nuts with a similar spectral profile, i.e. the absorbance peak at $1200 \mathrm{~nm}$, related to long chain fatty acids. This property is well adapted to the prevention of food allergies due to nut contaminations. In general, the population sensitive to one $\mathrm{TN}$ is also sensitive to other types of TNs and to peanuts, ${ }^{3}$ and the medical recommendation according to the previous research work on allergens recommends abstaining from ingestion of any nuts. ${ }^{4}$ Hence, in this present work it is not very important to be specific about the type of nuts detected but more to focus on the detection of different nuts in general for a multi-product processing environment.

Figure 4 represents the index values of the simulated hyperspectral image used for validation. As can be seen in the figure, a clear contrast was observed between nuts (peanut, walnut and hazelnut), with lower index values (more negative), represented in shades of blue and the background flour, with higher index values, represented in red shades.

\section{Behaviour of mixed spectra}

One of the challenges found in using imaging systems to detect product traces in any food products, especially in case of powder foods where the size is very small, is the presence of mixed pixels. Each pixel of the image corresponds to a small portion of the sample, for which a single spectrum is considered to be a linear combination of the different compounds present in the sample. Hence, when more than one product

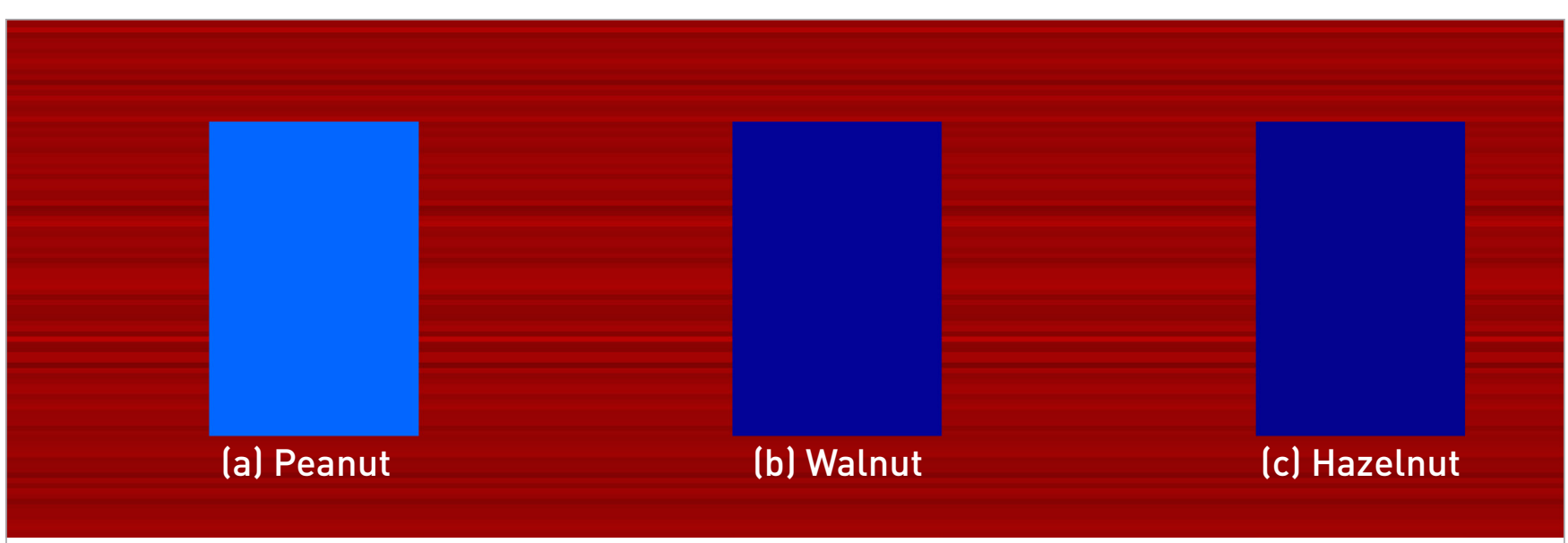

Figure 4. Validation score image $(100 \times 300)$ obtained after the application of the index. Three different blocks represents the different nuts (a) peanut, (b) walnut and (c) hazelnut. 
is found in the sample area corresponding to a particular pixel, the pixel spectra is "mixed". When the particle size of adulterants is similar or even smaller than the pixel size, it is particularly important to take this effect into account, as it would affect most of the pixels containing the adulterant product traces and so the accuracy of limit of detection. In this case, if only pure nut spectra were identified, the adulteration percentage of the samples would be considerably underestimated and also it would provide false negatives. According to this, considering the spectral profiles and index values of mixed spectra in relation to the threshold value could be used to act on the risk of false positive or negative errors.

To understand this, a simulation of the behaviour of mixed pixels is given in Figure 5. The simulation is performed considering pixels to be a linear combination of pure wheat flour and peanut spectra. The simulation was made with addition of peanut spectra to the wheat spectra in $20 \%$ increments until $100 \%$ peanut spectra are obtained. The corresponding index values for the mixed spectra after the application of the index are also indicated at the end of the spectra in Figure 5. From Figure 5, it can be seen that a pure pixel of peanut will be represented by the top-most spectra, providing an index value of -0.83 and a pure pixel of wheat flour by the bottom most spectra, providing an index value of -0.14 . Pure peanut possesses a higher absolute index value than pure wheat flour because of the high absorption peak (1200 nm) compared to wheat flour (Figure 2). Hence, their relative presence in a pixel will give an index value with in the range of -0.14 to -0.83 . The index values of mixed spectra lie in the range between the values for pure peanut and wheat flour $(-0.83$ and -0.14$)$.

\section{Peanut traces detection}

Coherently, to the work of Cuadrado et al., ${ }^{21}$ with detection of peanut traces irrespective of the roasting treatments, the index was successful to detect the presence of walnut and hazelnut traces in samples from $10 \%$ down to $0.01 \%$ adulteration. Figure 6 shows index images (first row) and segmented images (second row) obtained by the application of the SI and threshold on flour samples adulterated with $0.01 \%$ of peanut,

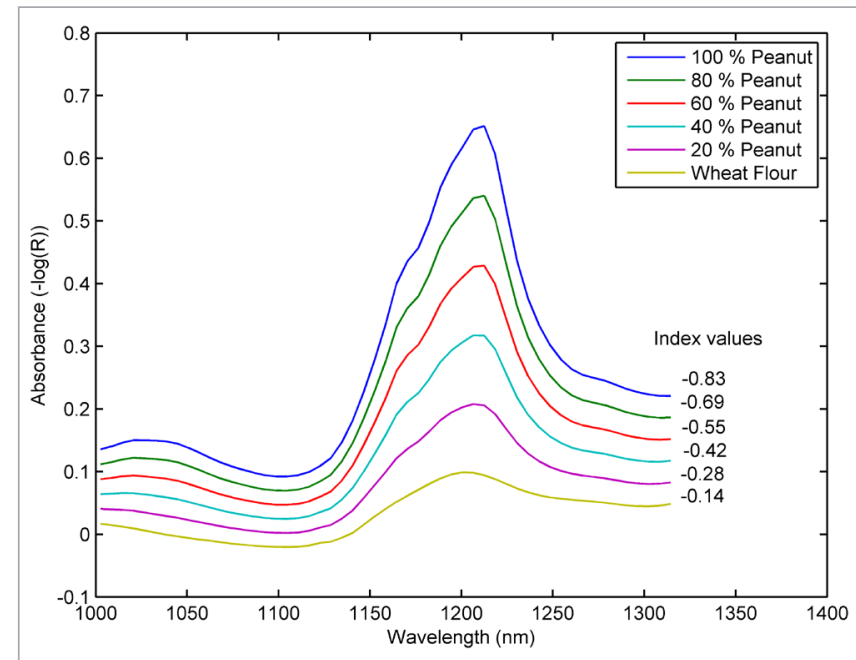

Figure 5. Simulation for the behaviour of a mixed pixel detected by the camera lexplained as a linear combination of wheat flour and peanut).

hazelnut and walnut seperately. The mentioned thresholds in Figure 6, used for different nuts, were obtained from the histogram obtained for the index images of pure wheat flour with different nuts separately. The amount of nut detected also depends on the actual amount of nut particles on the surface. For that concern, theoretical ranges of presence for different percentage presence have been defined in Table 1 with $95 \%$ confidence. These intervals have been computed taken into account the standard deviation resulting from the theoretical proportion of pixels and the number of pixels of the image. The data shown in the columns for "calculated nuts" correspond to the estimation of pixels belonging to nuts on the indexed images after threshold. It can be seen in the table that for $10 \%$ adulteration, peanut and walnut follow the range and at $0.01 \%$ adulteration, the range is followed by walnut and hazelnut. Amount of peanut was overestimated at trace levels below $0.5 \%$ adulteration. However, the detection of walnut and hazelnut was in the range below $0.5 \%$ adulteration. It should be noted that the experiment was not carried out in monolayer

Table 1. Trace detection results obtained after processing and thresholding the HSI images. Images of mix 1 to mix 8 for the three types of mixtures (i.e. peanut in flour, walnut in flour and hazelnut in flour separately) contain $130 \times 340$ lines and images of mix 7 and mix 8 contain $130 \times 600$ lines.

\begin{tabular}{|l|c|c|c|c|c|}
\hline Sample & $\begin{array}{c}\text { Actual } \\
\text { amount (\%) }\end{array}$ & $\begin{array}{c}\text { Range of theoretical } \\
\text { nuts present in flour } \\
(95 \% \text { confidence) }\end{array}$ & $\begin{array}{c}\text { Calculated peanut } \\
(\%)\end{array}$ & $\begin{array}{c}\text { Calculated walnut } \\
(\%)\end{array}$ & $\begin{array}{c}\text { Calculated } \\
\text { hazelnut (\%) }\end{array}$ \\
\hline Mix 1 & 10.00 & $9.714-10.2854$ & 9.72 & 9.96 & 9.46 \\
\hline Mix 2 & 5.00 & $4.793-5.207$ & 2.87 & 2.64 & 3.13 \\
\hline Mix 3 & 2.50 & $2.351-2.648$ & 2.12 & 1.58 & 1.02 \\
\hline Mix 4 & 1.00 & $0.905-1.094$ & 1.05 & 0.68 & 0.61 \\
\hline Mix 5 & 0.50 & $0.432-0.567$ & 0.39 & 0.48 & 0.24 \\
\hline Mix 6 & 0.10 & $0.069-0.130$ & 0.28 & 0.09 & 0.05 \\
\hline Mix 7 & 0.05 & $0.034-0.066$ & 0.19 & 0.04 & 0.11 \\
\hline Mix 8 & 0.01 & $0.003-0.017$ & 0.13 & 0.015 & 0.015 \\
\hline
\end{tabular}




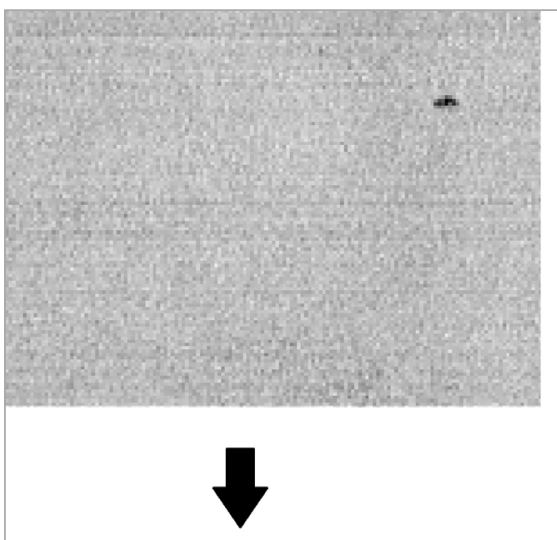

Threshold (-0.205)
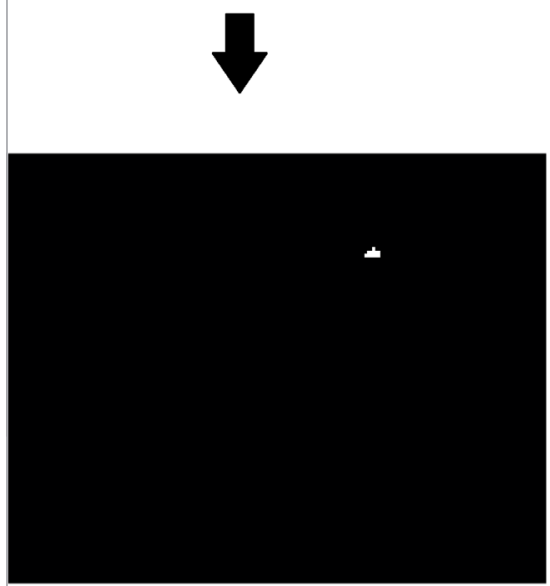

Walnut
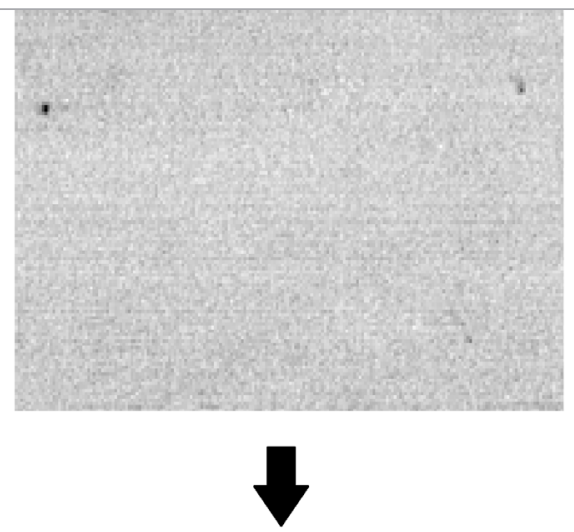

Threshold (-0.210)

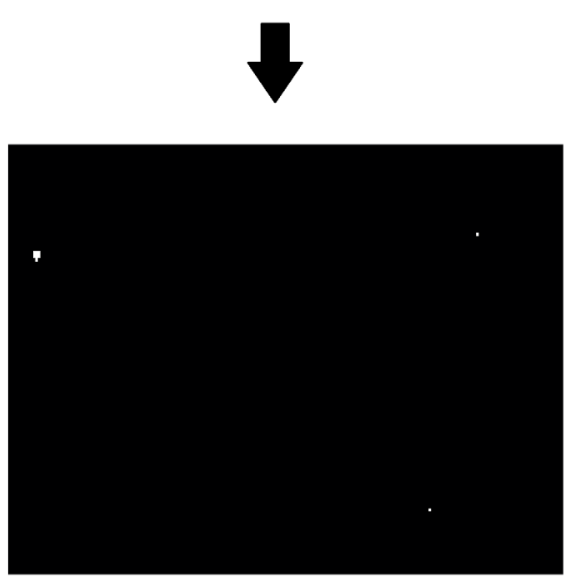

Hazelnut
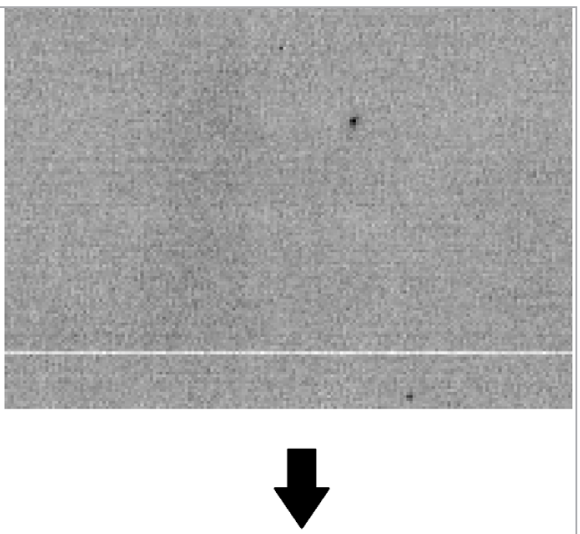

Threshold (-0.190)
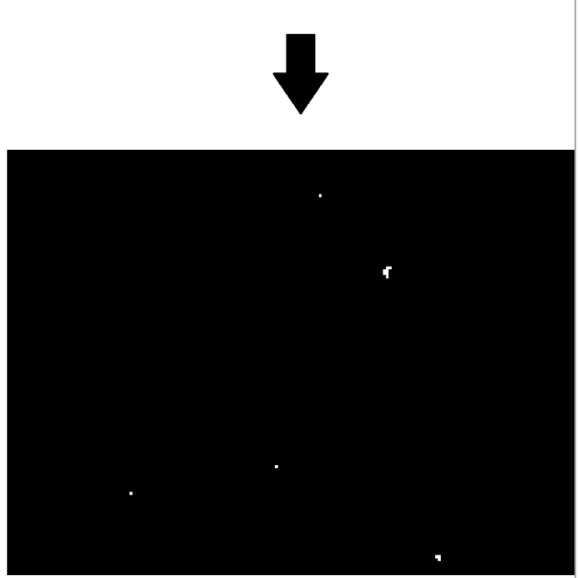

Peanut

Figure 6. Index and Segmented Index images obtained after the application of the selected thresholds for $0.01 \%$ of peanut, walnut and hazelnut.

distribution of sample for image acquisition and hence the amount of nuts present on the surface can also vary much more than is shown under the range of $95 \%$ confidence. To deal with estimation accuracy, more work is needed to repeat the analysis with new samples and to perform imaging in different technical conditions. The aim of this work was limited to the detection of nut traces as compared to the estimation of adulteration.

Figure 6, second row shows the segmented images obtained by the application of the SI. As shown in Figure 2, peanut traces could be detected in samples from 10\% down to $0.01 \%$ adulteration. In the case of peanut we can see that there are some false positives, which are well understood from the results of overestimation shown in Table 1. To deal with this issue, further study of mixed pixels and their effect on determining the threshold is needed.

Selectivity of different wavebands is crucial for designing a multispectral system. After designing the multispectral imaging system, various factors such as detection limit, accuracy and the sensitivity of detection of different products simultaneously defines the feasibility of the system. The spectral index discussed in this work with the detection of products, also, helps in improving the quality of the result. The index used in this study provides an improvement of the result by simultaneously removing additive effects from the image. The index is the estimation of the second derivative and the second order differential, and is sufficient for the removal of the additive effect. In spectral imaging, the additive effect has a major effect on deciding the quality of the final results. These effects are most common when the surface is not homogeneous, as the surface inhomogeneity leading to such an effect will diffract the light entering the surface. In our case, the surface scanned by the camera is not homogenous, since the sample is in powder form and contains small particles which can act as diffractors for the incoming light. In this case an index, which is also an estimate of the second derivative, can be usefully implemented as it will reduce the effect of light scattering and provide more accurate results.

A multispectral system is efficient if it can detect different similar compounds under the same experimental setup and technical configurations. Nevertheless, multispectral imaging for the assessment of adulterated products faces some additional challenges compared to point contact spectroscopy of pure samples. ${ }^{21,22}$ The choice of illumination and sample presentation have a greater impact on the measurements, as they are taken at a greater distance from the samples and product 
presentation is not standardised. In addition, the detection of small traces of ground products in a matrix of powder food, presents several imaging-specific problems, such as the presence of mixed pixels, containing both nuts and wheat flour spectra, and the image segmentation process needed to identify the adulterant particles in the image. More work is needed to deal with different flour products such as soy, semolina etc. Also, future work will be focused on specificity and selectivity to detect tree nuts and peanuts when present together.

\section{Conclusion}

The application of the spectral index on corresponding spectral planes extracted from hyperspectral images showed promising results when used for the detection of tree nuts and peanut adulteration in wheat flour samples. It showed good performance in detecting traces down to $0.01 \%$ by weight. The spectral index provides an estimate of second derivative, which helped in dealing with the effects arising from light scattering to further enhance the detection of traces in the wheat flour.

This work represents a further step towards the implementation of more reliable, cheap and practical methods for the detection of tree nuts and peanut adulteration in powder foods, regardless of whether the adulteration is intentional or a mistake in product handling during processing in a multiproduct processing environment. The spatial information provided by the multispectral images facilitates the representative sampling of the powder products. In addition, the measurement procedure can be adapted to take in-line measurements in processing lines. In this context, multispectral imaging could operate in conjuction with chemical procedures, such as RT-PCR and ELISA. This approach could allow not only a few selected samples to be assessed but also to incorporate extensive quality control surveyance in product processing lines while saving the time, money and skilled labour required.

\section{Acknowledgements}

The authors are grateful for peanut sample sets used in the work provided by the European Commission Joint Research Centre, Institute for Reference Materials and Measurements. We also gratefully acknowledge the technical assistance provided by Daniel Moura and Ryad Bendoula (IRSTEA, UMR ITAP Montpellier) in our research work.

\section{References}

1. J. Gray, "Nuts and seeds", in Encyclopedia of Human Nutrition (Third Edn), Ed by B. Caballero. Academic Press, Waltham, pp. 329-335 (2013). doi: http://dx.doi. org/10.1016/B978-0-12-375083-9.00208-7

2. M.M. Cochard and P.A. Eigenmann, "Allergies to nuts and seeds", in Nuts and Seeds in Health and Disease Prevention, Ed by V.R. Preedy, R.R. Watson and V.B. Patel.
Academic Press, San Diego, pp. 137-143 (2011). doi: http://dx.doi.org/10.1016/B978-0-12-375688-6.10015-5

3. M.P. de Leon, A.C. Drew, I.N. Glaspole, C. Suphioglu, R.E. O'Hehir and J.M. Rolland, "IgE cross-reactivity between the major peanut allergen Ara h 2 and tree nut allergens", Mol. Immunol. 44(4), 463-471 (2007). doi: http:// dx.doi.org/10.1016/j.molimm.2006.02.016

4. A. Barre, C. Sordet, R. Culerrier, F. Rancé, A. Didier and P. Rougé, "Vicilin allergens of peanut and tree nuts (walnut, hazelnut and cashew nut) share structurally related IgE-binding epitopes", Mol. Immunol. 45(5), 1231-1240 (2008). doi: http://dx.doi.org/10.1016/j. molimm.2007.09.014

5. D.M. Fleischer, M.K. Conover-Walker, E.C. Matsui and R.A. Wood, "The natural history of tree nut allergy", J. Allergy Clin. Immunol. 116(5), 1087-1093 (2005). doi: http://dx.doi.org/10.1016/i.jaci.2005.09.002

6. L.N. Willison, S.K. Sathe and K.H. Roux, "Production and analysis of recombinant tree nut allergens", Methods 66(1), 34-43 (2014). doi: http://dx.doi.org/10.1016/j. ymeth.2013.07.033

7. M. Kulis, Y. Li, H. Lane, L. Pons and W. Burks, "Singletree nut immunotherapy attenuates allergic reactions in mice with hypersensitivity to multiple tree nuts", J. Allergy Clin. Immunol. 127(1), 81-88 (2011). doi: http:// dx.doi.org/10.1016/i.jaci.2010.09.014

8. C. Platteau, M. De Loose, B. De Meulenaer and I. Taverniers, “Detection of allergenic ingredients using real-time PCR: a case study on hazelnut (Corylus avellanal and soy (Glicine max)", J. Agric. Food Chem. 59, 10803-10814 (2011). doi: http://dx.doi.org/10.1021/ if202110f

9. I.M. López-Calleja, S. de la Cruz, N. Pegels, I. González, T. García and R. Martín, “Development of a real time PCR assay for detection of allergenic trace amounts of peanut (Arachis hypogaea) in processed foods", Food Control 30, 480-490 (2013). doi: http://dx.doi.org/10.1016/j.foodcont.2012.09.017

10. T.L. Hostetler, S.G. Hostetler, G. Phillips and B.L. Martin, "The ability of adults and children to visually identify peanuts and tree nuts", Ann. Allerg. Asthma Immunol. 108(1), 25-29 (2012). doi: http://dx.doi.org/10.1016/j. anai.2011.09.012

11. V. Chelladurai, K. Karuppiah, D.S. Jayas, P.G. Fields and N.D.G. White, "Detection of Callosobruchus maculatus (F.) infestation in soybean using soft X-ray and NIR hyperspectral imaging techniques", J. Stored Prod. Res. 57, 43-48 (2014). doi: http://dx.doi.org/10.1016/j. jspr.2013.12.005

12. X. Fu, M.S. Kim, K. Chao, J. Qin, J. Lim, H. Lee, A. Garrido-Varo, D. Pérez-Marín and Y. Ying, "Detection of melamine in milk powders based on NIR hyperspectral imaging and spectral similarity analyses", J. Food Eng.

124, 97-104 (2014). doi: http://dx.doi.org/10.1016/j.jfoodeng.2013.09.023

13. J.A.F. Pierna, D. Vincke, P. Dardenne, Z. Yang, L. Hanb and V. Baeten, "Line scan hyperspectral imaging spectroscopy for the early detection of melamine and cyanu- 
ric acid in feed", J. Near Infrared Spectrosc. 22, 103-112 (2014). doi: http://dx.doi.org/10.1255/jnirs.1109

14. J. Qin, K. Chao, M.S. Kim, R. Lu and T.F. Burks, "Hyperspectral and multispectral imaging for evaluating food safety and quality", J. Food Eng. 118(2), 157-171 (2013). doi: http://dx.doi.org/10.1016/j.jfoodeng.2013.04.001

15. B. Diezma, L. Lleó, J.M. Roger, A. Herrero, L. Lunadei and M. Ruiz-Altisent, "Examination of the quality of spinach leaves using hyperspectral imaging", Postharvest Biol. Technol. 85, 8-17 (2013). doi: http://dx.doi. org/10.1016/j.postharvbio.2013.04.017

16. L. Lleó, J.M. Roger, A. Herrero-Langreo, B. DiezmaIglesias and P. Barreiro, "Comparison of multispectral indexes extracted from hyperspectral images for the assessment of fruit ripening", J. Food Eng. 104(4), 612-620 (2011). doi: http://dx.doi.org/10.1016/j.jfoodeng.2011.01.028

17. C. Liu, W. Liu, X. Lu, W. Chen, J. Yang and L. Zheng, "Nondestructive determination of transgenic Bacillus thuringiensis rice seeds (Oryza sativa L.) using multispectral imaging and chemometric methods", Food Chem. 153, 87-93 (2014). doi: http://dx.doi.org/10.1016/j. foodchem.2013.11.166

18. F. Ma, J. Yao, T. Xie, C. Liu, W. Chen, C. Chen and L. Zheng, "Multispectral imaging for rapid and nondestructive determination of aerobic plate count (APC) in cooked pork sausages", Food Res. Int. 62, 902-908 (2014). doi: http://dx.doi.org/10.1016/j.foodres.2014.05.010
19. H. Irshad, A. Gouaillard, L. Roux and D. Racoceanu, "Multispectral band selection and spatial characterization: Application to mitosis detection in breast cancer histopathology", Comput. Med. Imag. Grap. 38(5), 390402 (2014). doi: http://dx.doi.org/10.1016/i.compmedimag. 2014.04.003

20. C. Yang, M.S. Kim, P. Millner, K. Chao, B. Cho, C. Mo, H. Lee and D.E. Chan, "Development of multispectral imaging algorithm for detection of frass on mature red tomatoes", Postharvest Biol. Technol. 93, 1-8 (2014). doi: http://dx.doi.org/10.1016/j.postharvbio.2014.01.022

21. T. Cuadrado, S. Ghosh, P. Barreiro, B. Diezma and L. Lleó, Empleo de la Espectroscopia VIS NIR para la Identificación de Trazas de Cacahuete en Productos Alimentarios en Polvo. VII Congreso Ibérico Agroingeniería y Ciencias Hortícolas, Madrid, 26-29 Septiembre 2013.

22. S. Ghosh, T.R. Cuadrado, B. Diezma, L. Lleó, P. Barreiro, T.G. Lacarra and J.M. Roger, "VIS/NIR spectral signature of reference peanut powder samples for food ingredient discrimination", Agric. Eng. Int.: CIGR J. (in press) (2015).

23. S. Piqueras, J. Burger, R. Tauler and A. De Juan, "Relevant aspects of quantification and sample heterogeneity in hyperspectral image resolution", Chemometr. Intell. Lab. Syst. 117, 169-182 (2012). doi: http://dx.doi. org/10.1016/j.chemolab.2011.12.004

24. C.L. Tsai, J.C. Chen and W.J. Wang, "Near-infrared absorption property of biological soft tissue constituents", J. Med. Biol. Eng. 21, 7-14 (2001). 